\title{
Pilot-Aided Equalization with a Constrained Noise-Estimation Filter
}

\author{
M. Magarini, A. Spalvieri, L. Barletta \\ Dipartimento di Elettronica e Informazione \\ Politecnico di Milano \\ Milano, Italy \\ Email: \{magarini,spalvier,barletta\}@elet.polimi.it
}

\begin{abstract}
In this paper we focus on a single carrier pilotassisted transmission scheme where one pilot symbol is periodically inserted in the transmitted sequence on a time-division multiplexing basis. A new equalization scheme, where the knowledge of pilot symbols is exploited by the equalizer to generate an estimate of the noise affecting the symbol to be detected, is introduced and analyzed. The criterion used to compute the equalizer coefficients is the minimization of the mean-square error (MSE). The main new result of our analysis is that the optimal pilot aided equalizer (PAE) can be decomposed as the cascade of an unconstrained minimum MSE (MMSE) linear equalizer (LE) and a data-aided noise estimation filter. This result completes and extends the noise-predictive view of decision feedback equalization to general data-aided equalization. The PAE is compared here to the MMSE-LE and to the MSE decision feedback equalizer on two frequency selective wireless channels.
\end{abstract}

\section{INTRODUCTION}

The use of pilot aided transmission (PAT) is common to many wireless communication systems, see [1] and references therein. In this work we introduce a new time-domain equalization scheme for single-carrier (SC) PAT schemes where one known pilot symbol is time-division multiplexed with the random information symbols. Like pilot tones in OFDM, pilot symbols in SC are usually exploited by training-based estimators to improve the quality of channel estimation, synchronization and receiver adaptation. However, systems that make use of known pilot symbols to improve equalizer's performance are less studied.

To exploit the knowledge of pilot symbols, the equalizer can remove the interference they induce on the decision variable that is present at the detector's input. When such an intersymbol interference (ISI) cancellation approach is adopted for channel equalization, the receiver consists of a pre-filter and of a data-aided ISI cancellation filter, as shown by Mueller and Salz in [2]. The task of the ISI cancellation filter is to generate an estimate of the ISI and to subtract it from the signal at the output of the pre-filter. If the ISI were perfectly estimated, the optimum pre-filter would be the matched filter (MF) and the best possible performance would be achieved. Based on the general theory developed by Mueller and Salz, Gersho and Lim proposed in [3] to use causal and non-causal decisions to improve the performance over the ISI channel.

In this paper we propose an equalization scheme where such perfect estimates rely on the known pilot symbols. We propose and analyze a pilot aided equalizer (PAE) based on a noiseestimation approach as an alternative to the ISI cancellation approach. Estimation is based on the knowledge of noise and residual ISI at the pilot instants. The number of pilot symbols used by the noise-estimation filter can be constrained or unconstrained. The case of the unconstrained noise-estimation filter has been considered in [4]. In this paper we present the analysis for the constrained estimator. Our main result compared to [2] is that the optimization of the noise-estimation filter can be carried out independently of the optimization of the pre-filter, provided that the pre-filter is unconstrained. In a sense, this result extends the result of Belfiore and Park about the optimality of the noise-predictive decision feedback equalizer (DFE) [5]. For the given time-division multiplexing (TDM) frame of the pilot-symbols we show that the MSE turns out to be cyclostationary with period equal to $M$, where $M$ is the time spacing between two successive pilot symbols. The transfer function of the pre-filter is time-invariant and is the cascade of the MF and of the linear minimum MSE (LMMSE) equalizer. The optimal responses of the $M$ noise-estimation filters, one for each phase of the period, is obtained by using Wiener's method.

The paper is organized as follows. The system model of the TDM-PAT system we focus on is defined in section II. The derivation of the pre-filter and noise-estimation filter based on the MSE criterion is given in section III. Experimental results are shown in section IV, while conclusions are drawn in section $\mathrm{V}$.

\section{SYSTEM MODEL}

The time-discrete additive white Gaussian noise (AWGN) channel model is adopted to represent the received signal

$$
r[k]=\sum_{l=-\infty}^{\infty} d[l] g[k-l]+w[k]
$$

where $g[k]$ is the known impulse response of the channel, $w[k]$ is the random sequence of complex white Gaussian noise with power spectral density $N_{0}$, and $d[k]$ is the sequence that includes payload symbols and pilot symbols. In the following we assume that $d[k]$ is a sequence of i.i.d. random variables with zero mean and unit variance for $k \neq i M$, and that the $i$-th pilot symbol occurs at time $k=i M$. The bandwidth efficiency 


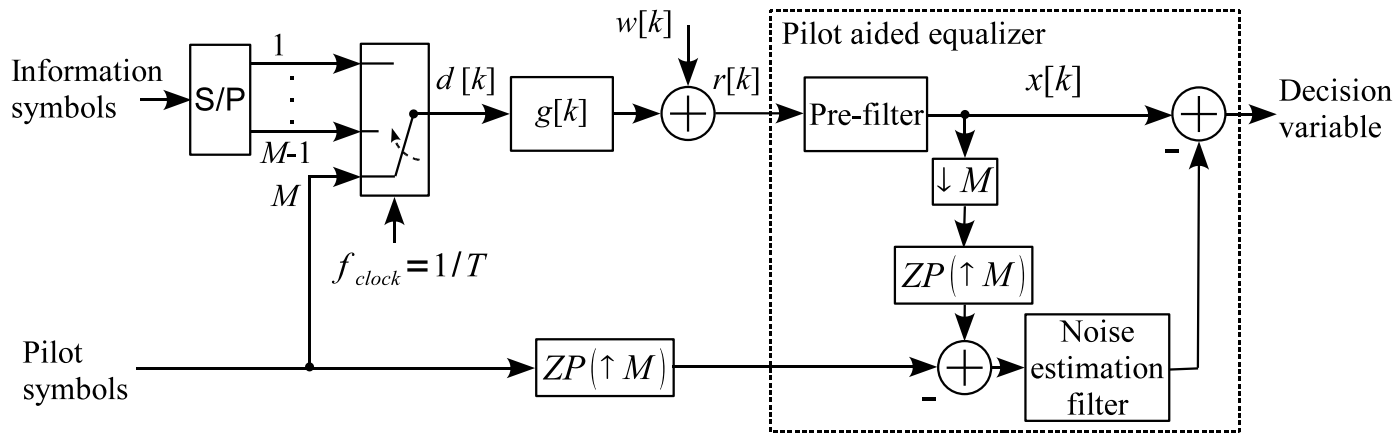

Fig. 1. Block diagram of the transmission system and of the PAE. The serial-to-parallel (S/P) block converts the serial data stream into a block of $M-1$ parallel symbols that are combined with the pilot symbol and subsequently parallel-to-serial converted to be transmitted at the symbol frequency $1 / T$. The block ZP $(\uparrow M)$ appends $M-1$ zeros after one valid sample (up-sampling with zero-padding). The block $\downarrow M$ performs decimation by a factor $M$ to extract samples in the pilot symbol instants.

resulting from the TDM structure of the pilot symbols is $1-$ $1 / M$.

The block diagram of the transmission system is shown in Figure 1. The received sequence (1) is filtered by the pre-filter to produce the sequence

$$
x[k]=d[k]+n[k],
$$

where $n[k]$ is the sum of Gaussian noise and ISI. The pilot symbols are used to compute the sequence

$$
n[i M]=x[i M]-d[i M] .
$$

In order to derive the optimum pre-filter and the optimum noise-estimation filter we adopt the noise-estimation approach. According to such an approach, the role of the noiseestimation filter is that of filtering the zero-padded sequence $n[i M]$ to produce an estimate of the disturbance $n[k]$ that affects $x[k]$. Then the estimate is subtracted from $x[k]$ to improve the performance. Since the sequence $n[i M]$ is cyclostationary with period $M$, then the output of the noiseestimation filter will be cyclostationary with period $M$ as well. In what follows the noise-estimate is denoted as

$$
\hat{n}[i M+m],
$$

and the range

$$
m=1,2, \cdots, M
$$

is considered. Also, in what follows, index $m$ is used uniquely to indicate the $M$ phases of the period, and, everywhere $m$ occurs, it is understood that only the values of $m$ of (3) are to be considered.

Assuming that the same number of pilots is used to construct the estimate in all the phases of the cycle, the estimate of the noise plus ISI sequence produced by the noise estimation filter at the $m$-th phase of the cycle is

$$
\hat{n}[k M+m]=\sum_{i=-\delta_{c}}^{\delta_{n}} n[i M] b[k M+m-i M],
$$

where $b[k]$ is the impulse response of the noise-estimation filter, and $\delta_{n}+\delta_{c}+1$ pilots are used to construct the estimate.
The impulse response $\{b[i]\}$ of the estimation filter is zero for $i \leq-\delta_{n} M$ and for $i>\left(\delta_{c}+1\right) M$. The parameter $\delta_{n}$ indicates the number of non-causal pilots used in the estimation, while $\delta_{c}+1$ is the number of strictly causal pilots. When $\delta_{n}=0$ the estimate is 1-causal for all the $M$ phases. For concreteness, one can bring in mind that the input samples $\left(n\left[-\delta_{c} M\right], \cdots, n[0], \cdots, n\left[\delta_{n} M\right]\right)$ are used to determine the estimate $\hat{n}[m], m=1,2, \cdots, M$.

\section{OPTIMIZATION OF THE PRE-FILTER AND OF THE NOISE-ESTIMATION FILTER}

The criterion that we adopt to optimize the pre-filter and the noise-estimation filter is the minimization of the MSE. The cyclostationary MSE at the $m$-th cycle of the period is

$$
\mathrm{MSE}_{m}=E\left\{|n[m+i M]-\hat{n}[m+i M]|^{2}\right\},
$$

where $E\{\cdot\}$ denotes the expected value.

\section{A. Optimal pre-filter}

Let the frequency response of the $m$-th noise-estimation filter be

$$
B_{m}(f)=\sum_{i=-\delta_{n}}^{\delta_{c}} b[m+i M] e^{-j 2 \pi(m+i M) f T} .
$$

The MSE given in (5) is computed as

$$
\operatorname{MSE}_{m}=T \int_{0}^{T^{-1}} \Psi(f)\left|1-B_{m}(f)\right|^{2} d f
$$

where $T$ is the symbol repetition interval and $\Psi(f)$ is the power spectral density of $n[k]$. Since $\Psi(f) \geq 0$, we see from (6) that, for any fixed $B_{m}(f)$, the optimal pre-filter is the one that minimizes $\Psi(f)$ frequency-by-frequency. Hence the optimal pre-filter is the minimum MSE linear equalizer (MMSE-LE), whose frequency response is

$$
\frac{G^{*}(f)}{|G(f)|^{2}+N_{0}}
$$


where $G(f)$ is the Fourier transform of $g[k]$. The power spectral density of ISI plus Gaussian noise after the MMSELE pre-filter is

$$
\Psi(f)=\frac{N_{0}}{|G(f)|^{2}+N_{0}} .
$$

\section{B. Noise-estimation filter}

Let the $k$-th sample of the autocorrelation of the noise be

$$
\psi[k]=E\left\{n^{*}[i] n[i+k]\right\},
$$

and organize the samples of the autocorrelation of the noise in the $M$ column vectors

$$
\boldsymbol{\psi}_{m}=\left(\psi\left[-\delta_{n} M+m\right], \cdots, \psi[m], \cdots, \psi\left[\delta_{c} M+m\right]\right)^{T},
$$

where the superscript ${ }^{T}$ denotes transposition. Let

$$
\boldsymbol{\Psi}=\left(\begin{array}{cccc}
\psi[0] & \psi[-M] & \cdots & \psi[-\delta M] \\
\psi[M] & \psi[0] & \cdots & \psi[(-\delta+1) M] \\
\vdots & \vdots & \ddots & \vdots \\
\psi[\delta M] & \psi[(\delta-1) M] & \cdots & \psi[0]
\end{array}\right)
$$

be the autocorrelation matrix of the noise sampled at pilot rate, with $\delta=\delta_{c}+\delta_{n}$. Invoking the orthogonality principle, one finds that the $m$-th optimal noise-estimation filter is the solution to the linear system

$$
\boldsymbol{\Psi} \mathbf{b}_{m}=\boldsymbol{\psi}_{m},
$$

where the impulse response of the $m$-th noise-estimation filter is the column vector

$$
\mathbf{b}_{m}=\left(b\left[-\delta_{n} M+m\right], \cdots, b[m], \cdots, b\left[\delta_{c} M+m\right]\right)^{T} .
$$

Provided that $\Psi$ is invertible, the impulse response of the $m$-th optimal noise-estimation filter is

$$
\mathbf{b}_{m}=\Psi^{-1} \boldsymbol{\psi}_{m} .
$$

For the MSE one gets

$$
\mathrm{MSE}_{m}=E\left\{|n(m)|^{2}\right\}-\boldsymbol{\psi}_{m}^{H} \boldsymbol{\Psi}^{-1} \boldsymbol{\psi}_{m},
$$

where the superscript ${ }^{H}$ denotes the Hermitian transposed.

Two special cases of the optimal noise-estimation filter are hereafter pointed out. When $\delta_{n} \geq 1$, it happens that $\boldsymbol{\psi}_{M}$ is equal to the column in position $\delta_{n}$ of the autocorrelation matrix, hence, from equation (8), one realizes that $\mathbf{b}_{M}$ should select that column. To do this, the entry in position $\delta_{n}$ of vector $\mathbf{b}_{M}$ is 1 , while all the other entries of $\mathbf{b}_{M}$ are zero. Substituting the $\mathbf{b}_{M}$ just described in place of the product $\Psi^{-1} \boldsymbol{\psi}_{M}$ appearing in (10) one recognizes that $\mathrm{MSE}_{M}=0$, as it should be since the noise is known at the time instants $[i M]$ where pilot symbols are placed. Also note that, for $\delta_{n}=0$ and $M=1$ the cycle has only one phase, and the 1-causal noiseestimation filter given by (9) is the noise-predictive decision feedback equalizer of Belfiore and Park [5].

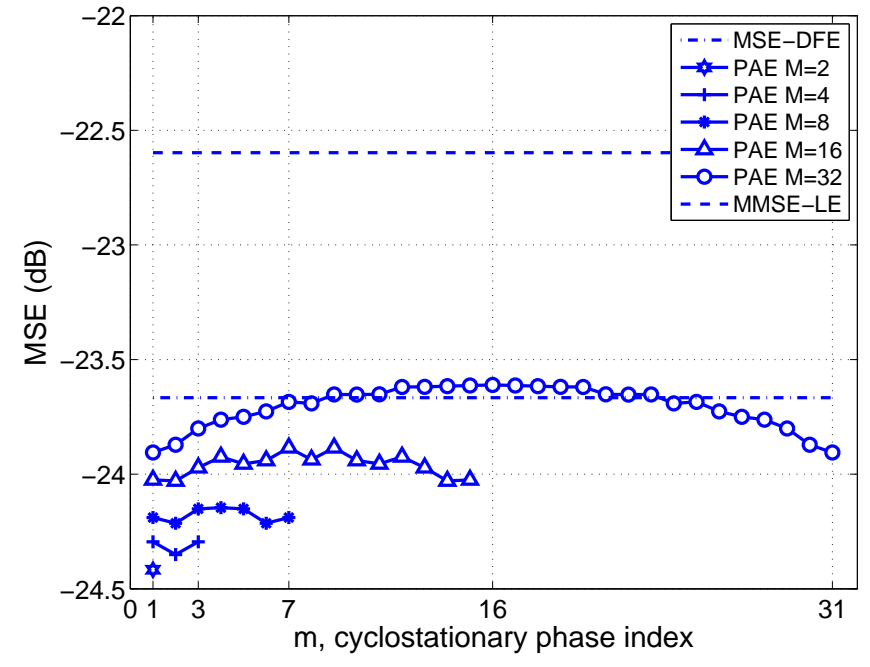

Fig. 2. Cyclostationary MSE for the Hiperlan type E channel at $\mathrm{SNR}=30 \mathrm{~dB}$ for a PAE with a noise-estimation filter with $\delta_{n}=1$ and $\delta_{c}=0$. The performance of the MMSE-LE and of the MSE-DFE with a feedback filter with 2 taps is also reported.

\section{EXPERIMENTAL RESULTS}

The performance of the proposed PAE with constrained noise-estimation filter is compared to the performance of the MSE decision feedback equalizer (MSE-DFE) with feedback filter having the same number of taps as the PAE and to the performance of the MMSE-LE. It is worth noting that, in what follows, we limit our analysis to the case where no decision errors affect the DFE, the effect of decision errors that may affect the DFE being left to future study. Simulation results are reported for two examples of transmission over dispersive multipath wireless channels. In the following we assume that the channel is always perfectly known to the receiver and that the transmit filter is a square root Nyquist filter with rolloff factor 0.25 . The shaped signal is passed through the multipath fading channel, filtered by a receive filter matched to the transmit filter and sampled at the symbol-rate frequency. The symbol rate of $20 \mathrm{MHz}$ is considered. The signal-to-noise ratio (SNR) is defined as the ratio between the average power of the useful signal to the variance of the noise at the sampling instant.

As a first example we consider the fading channel model E defined by ETSI BRAN for HiperLAN/2 [8]. A noiseestimation filter with $\delta_{n}=1, \delta_{c}=0$, that is with two taps, is considered. Simulations are used to generate $10^{4}$ realizations of the fading channel. For each channel realization the instantaneous $\mathrm{MSE}_{m}, m=1, \ldots, M$, is computed according to (10). For the MMSE-LE the MSE has been computed considering (7) as the sole integrand in (6), while for the MSE-DFE equation (65) of [5] has been used. The average $\mathrm{MSE}_{m}$ values for the considered channel are shown in Figure 2 for different $M \mathrm{~s}$ and $\mathrm{SNR}=30 \mathrm{~dB}$. For the considered values of $M$ we observe that the PAE allows to achieve a performance that is almost always better than that of the MSE-DFE. The improvement over the MSE-DFE is achieved 


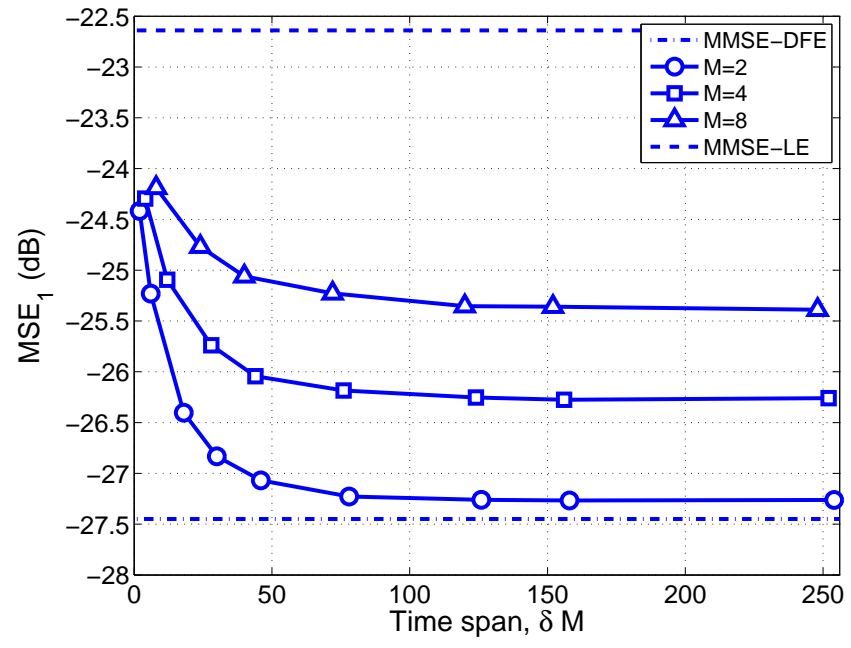

Fig. 3. MSE versus time span of the noise-estimation filter for the Hiperlan type $\mathrm{E}$ channel at $\mathrm{SNR}=30 \mathrm{~dB}$. The performance of the MMSE-LE and of the unconstrained MMSE-DFE is also reported.

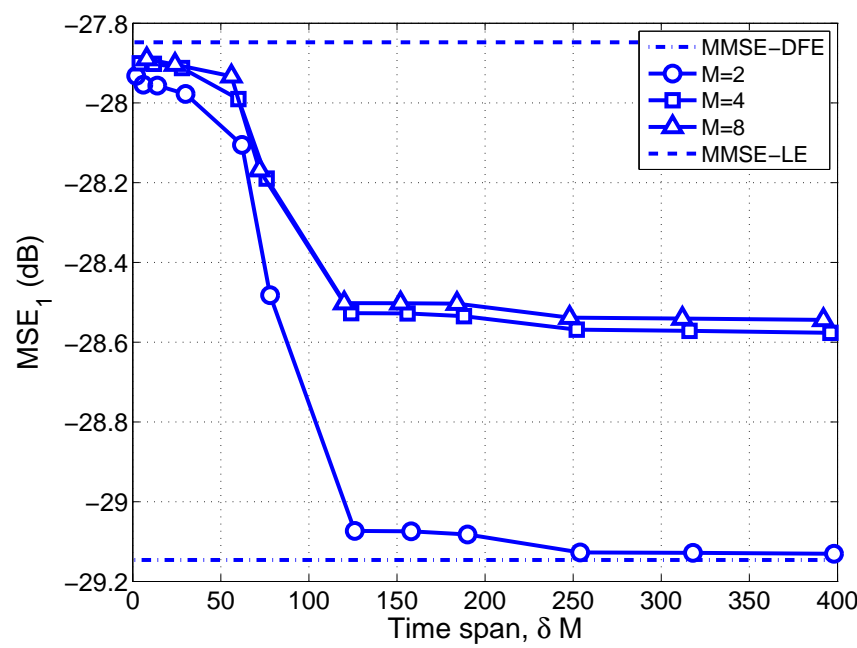

Fig. 4. MSE versus time span of the noise-estimation filter for the sparse channel of [7] at $\mathrm{SNR}=30 \mathrm{~dB}$. The performance of the MMSE-LE and of the unconstrained MMSE-DFE is also reported.

at the cost of a moderate reduction in bandwidth efficiency. However, we recall that the MSE of the MSE-DFE has been obtained under the ideal condition of absence of error propagation. We observe that when there is a constraint on the number of coefficients of the noise-estimation filter, as in the example shown in Figure 2, the PAE can potentially provide an advantage in terms of performance over the MSEDFE with only a small penalty in terms of spectral efficiency. The performance of the MMSE-LE is also reported in the Figure. Figure 3 reports the $\mathrm{MSE}_{1}$ performance, versus the time span $\delta M$ for $M=2,4,8$ at $\mathrm{SNR}=30 \mathrm{~dB}$. From the Figure we observe that for $M=2$ and $\delta M>80$ the performance is similar to that of the ideal MMSE-DFE with unconstrained noise-estimation filter.

As a second channel we consider the highly dispersive

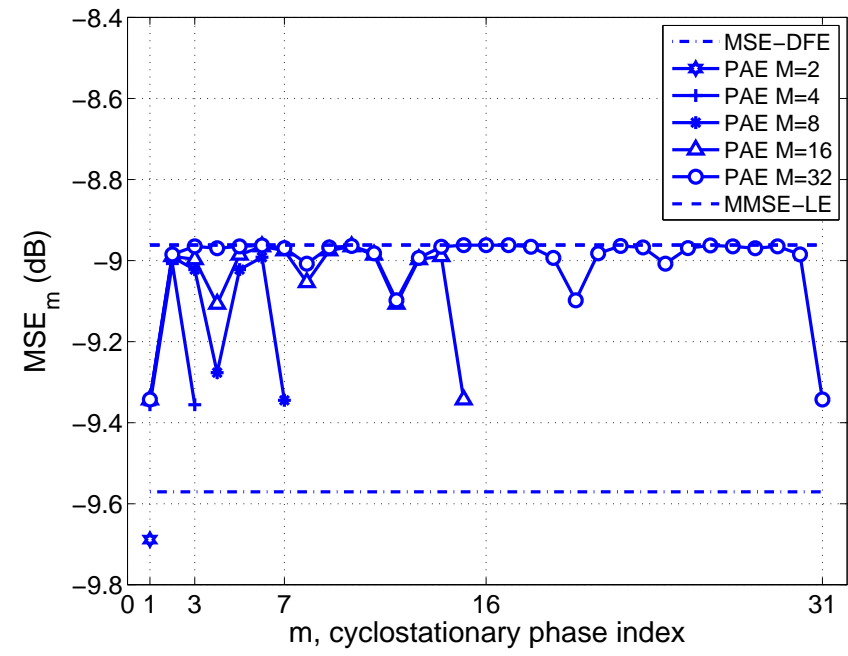

Fig. 5. Cyclostationary MSE for the sparse channel of [7] at $\mathrm{SNR}=10 \mathrm{~dB}$ for a PAE with a noise-estimation filter with $\delta_{n}=32$ and $\delta_{c}=31$ The performance of the MMSE-LE and of the MSE-DFE with a feedback filter with 64 taps is also reported.

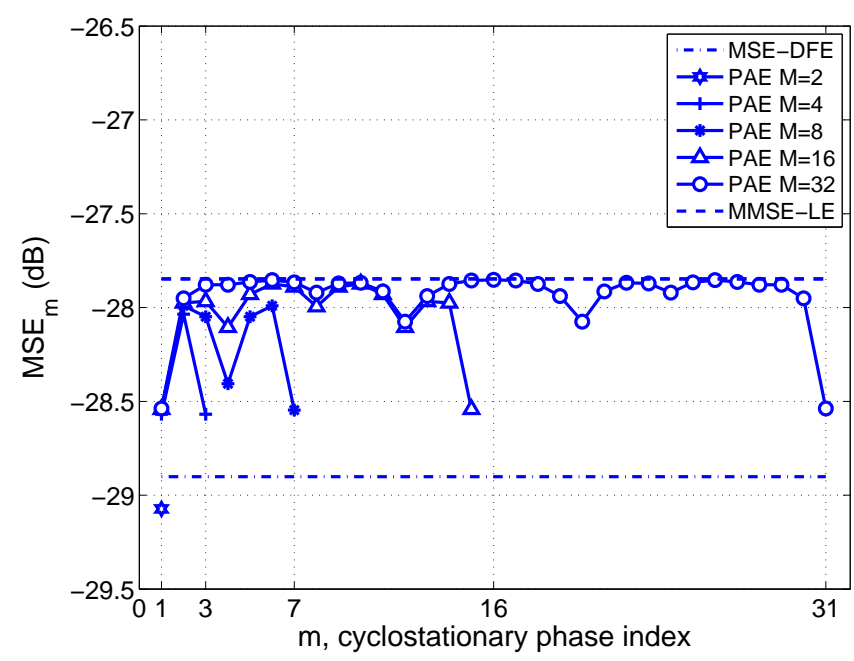

Fig. 6. Cyclostationary MSE for the sparse channel of [7] at $\mathrm{SNR}=30 \mathrm{~dB}$ for a PAE with a noise-estimation filter with $\delta_{n}=\delta_{c}=32$. The performance of the MMSE-LE and of the MSE-DFE with a feedback filter with 64 taps is also reported.

channel of [7]. This is a sparse multipath channel that can be used as a channel model for digital communication systems such as terrestrial broadcasting and cellular land mobile. The impulse response of the channel is characterized by a main echo, two anti-causal and three causal components, two of which are closely spaced. The duration of the channel is in the order of hundreds of symbol intervals, although the majority of taps in the sampled channel are near zero-valued. In Figure 4 the $\mathrm{MSE}_{1}$ performance of the PAE versus the time span $\delta M$ is shown for $M=2,4,8$ at $\mathrm{SNR}=30 \mathrm{~dB}$. In this case we observe that the difference between the MMSELE and the unconstrained MMSE-DFE is moderate. Moreover, one realizes that the PAE allows to reach the performance over the unconstrained MMSE-DFE only for $\delta M>128$ and 
$M=2$. However, the cost in terms of spectral efficiency is huge. The cyclostationary $\mathrm{MSE}_{m} \mathrm{~s}$ achieved by the PAE for different values of $M$ with $\delta_{n}=32, \delta_{c}=31$, that is a PAE with 64 taps independently of $M$, for $\mathrm{SNR}=10$ and $30 \mathrm{~dB}$ are shown in Figures 5 and 6, respectively. In these Figures the performance of the MMSE-LE and the performance of the MSE-DFE with 64 taps is also reported. These results confirm that the proposed system outperforms the DFE only with small $M$.

Note that to compute the bit error probability one should derive the probability density function of ISI plus noise, for example by the method proposed in [9]. A fairly accurate estimate is obtained by assuming that ISI and noise are independent Gaussian random variables. Specifically, by considering only the contribution of the errors between two closest points in the constellation, the bit error probability of an $L^{2}$ QAM constellation with Gray mapping can be approximated as

$$
P_{b}(e)=\frac{1}{M-1} \sum_{m=1}^{M-1} \frac{2(L-1)}{L \cdot \log _{2}(L)} Q\left(\sqrt{\frac{3\left(1-\mathrm{MSE}_{m}\right)}{\left(L^{2}-1\right) \mathrm{MSE}_{m}}}\right),
$$

where

$$
Q(x)=\frac{1}{\sqrt{2 \pi}} \int_{x}^{\infty} e^{-\frac{u^{2}}{2}} d u .
$$

We have verified that equation (11) closely fits simulation results for the channel models at hand at low-to-intermediate values of bit error probability.

\section{CONCLUSION}

In this paper a pilot-aided equalization scheme based on a noise-cancellation approach for single-carrier TDM pilot assisted transmission has been introduced and analyzed. The basic idea behind the proposed approach is that of cancelling the noise that affects the symbol to be detected by using an estimate of noise and residual ISI obtained by exploiting the knowledge of the pilot symbols. For the considered TDM structure of the pilot symbols we have shown that, when the MMSE is chosen as design criterion, the proposed pilot aided equalizer consists of an unconstrained MMSE-LE and a pilot-aided noise-estimation filter. The estimate of the noise produced at the output of the pilot-aided noise-estimation filter is subtracted from the signal at the output of the MMSE-LE to produce the decision variable for the detector. As shown by our numerical results, the MSE performance of the proposed pilot aided equalizer for the case of constrained noise-estimation filter can outperform, on certain channels, that of the MSEDFE with a feedback filter of the same length. It is worth noting that, in the proposed scheme, perfect estimate of the noise can be obtained from the knowledge of the pilot symbols at the cost of a reduction of information rate that is tolerable in many realistic cases. Therefore, compared to equalization methods based on tentative decisions, we can say that the PAE is a scheme that trades spectral efficiency with absence of error propagation.

\section{REFERENCES}

[1] L. Tong, B. M. Sadler, M. Dong, "Pilot-assisted wireless transmissions: general model, design criteria, and signal processing," IEEE Signal Proc. Mag., vol. 21, pp. 12-25, Nov. 2004.

[2] M. S. Mueller, J. Salz, "A unified theory of data-aided equalization," Bell Syst. Tech. J., vol. 60, pp. 2023-2038, Nov. 1981.

[3] A. Gersho, T. L. Lim, "Adaptive cancellation of intersymbol interference for data transmission," Bell Syst. Tech. J., vol. 60, pp. 1997-2021, Nov. 1981.

[4] L. G. Giordano, M. Magarini, A. Spalvieri, "Infinite-length pilot aided equalization,” IEEE Commun. Lett., vol. 14, pp. 465-467, May 2010.

[5] C. Belfiore, J. Park, "Decision feedback equalization," Proc. IEEE, vol. 67, pp. 1143-1156, Aug. 1979.

[6] J. G. Proakis, Digital communications, 3rd ed., Singapore: McGraw-Hill, 1995 , pp. 559.

[7] A. A. Rontogiannis, K. Berberidis, "Bandwidth efficient transmission through sparse channels using a parametric channel-estimation based DFE," Proc. IEE Proc.-Commun., vol. 152, pp. 251-256, Apr. 2005.

[8] J. Medbo, P. Schramm, "Channel models for HiperLAN2," ETSI/BRAN document no. 3ERI085B, 1998.

[9] J. C. Vanelli, N. M. Shehadeh, "Computation of bit error probability using the trapezoidal integration rule," IEEE Trans. Commun., vol. 22, pp. 331-334, March 1974. 